\title{
Agrobacterium-mediated vacuum infiltration and floral dip transformation of rapid- cycling Brassica rapa
}

\author{
Die $\mathrm{Hu}^{1,2}$, Andrew F. Bent ${ }^{2 *}$, Xilin $\mathrm{Hou}^{1}$ and Ying $\mathrm{Li}^{i^{*}}$
}

\begin{abstract}
Background: Rapid-cycling Brassica rapa (RCBr), also known as Wisconsin Fast Plants, are small robust plants with a short lifecycle that are widely used in biology teaching. RCBr have been used for decades but there are no published reports of RCBr genetic transformation. Agrobacterium-mediated vacuum infiltration has been used to transform pakchoi (Brassica rapa ssp. chinensis) and may be suitable for RCBr transformation. The floral dip transformation method, an improved version of vacuum infiltration, could make the procedure easier.

Results: Based on previous findings from Arabidopsis and pakchoi, plants of three different ages were inoculated with Agrobacterium. Kanamycin selection was suboptimal with RCBr; a GFP screen was used to identify candidate transformants. $\mathrm{RCBr}$ floral bud dissection showed that only buds with a diameter less than $1 \mathrm{~mm}$ carried unsealed carpels, a key point of successful floral dip transformation. Plants across a wide range of inflorescence maturities but containing these immature buds were successfully transformed, at an overall rate of $0.1 \%$ (one per $1000 \mathrm{~T}_{1}$ seeds). Transformation was successful using either vacuum infiltration or the floral dip method, as confirmed by PCR and Southern blot.
\end{abstract}

Conclusion: A genetic transformation system for $\mathrm{RCBr}$ was established in this study. This will promote development of new biology teaching tools as well as basic biology research on Brassica rapa.

Keywords: Rapid-cycling Brassica rapa, Wisconsin fast plants, Agrobacterium-mediated transformation, Vacuum infiltration, Floral dip

\section{Background}

Rapid-cycling Brassica rapa (RCBr), also known as Wisconsin Fast Plants, were derived from genetic crossing among multiple faster flowering Brassica rapa [1]. Because of this origin, $\mathrm{RCBr}$ inherited diverse traits. Equally important, the plants are petite and have a very short lifecycle of about 40 days if grown under continuous light. Due to these desirable characteristics, and the development of clever and inexpensive teaching modules by the Wisconsin Fast Plants program, $\mathrm{RCBr}$ have been used in thousands of classrooms to educate students about biology [2]. Scientists have also utilized $\mathrm{RCBr}$ for diverse

\footnotetext{
*Correspondence: afbent@wisc.edu; yingli@njau.edu.cn

${ }^{2}$ Department of Plant Pathology, University of Wisconsin-Madison, Madison, WI 53706, USA

${ }^{1}$ National Key Laboratory of Crop Genetics and Germplasm Enhancement, College of Horticulture, Nanjing Agricultural University, Nanjing 210095, Jiangsu Province, China
}

studies [3-7], and RCBr is a useful model for further study of Brassica rapa or of plants in general.

Genetic transformation is an important tool in molecular biology research and agricultural biotechnology application. For plants, Agrobacterium tumefaciens is widely used for gene delivery and tissue culture is commonly utilized to generate transformants. Brassica rapa is thought to be recalcitrant to in vitro shoot regeneration [8] and genetic transformation of Brassica rapa is challenging, requiring specialist approaches and yielding success at very low frequencies [9]. Genetic background differences among cultivars play an important role in Brassica rapa transformation efficiency [9]. Regeneration protocols for $\mathrm{RCBr}$ are available [10-12]. However, genetic transformation of $\mathrm{RCBr}$ has not been reported. Development of a transformation system would promote both basic research on Brassica rapa and enhanced use of $\mathrm{RCBr}$ as a teaching tool. 
In planta plant transformation methods, such as vacuum infiltration of Agrobacterium, are an alternative approach to plant transformation [13, 14]. Compared to tissue culture, such methods are typically less timeconsuming and labor-intensive [15]. Confirmed successes with in planta transformation had previously been limited to plants in the Brassicaceae, but successes have now been reported for Eustoma grandiflorum, Setaria viridis and Solanum lycopersicum [16-18]. Pakchoi (Brassica rapa ssp. chinensis, also called bok choy or non-heading Chinese cabbage) has been transformed via Agrobacterium-mediated vacuum infiltration method $[15,19]$, suggesting the feasibility of adopting the method for $\mathrm{RCBr}$ transformation. The floral dip method, an improved version of vacuum infiltration [20], has been widely adopted for transformation of Arabidopsis thaliana, offering a simple and inexpensive approach that has a high success rate. Because the vacuum infiltration process is unnecessary to transform Arabidopsis [14, 20], the viability of floral dip for transformation for the taxonomically related $\mathrm{RCBr}$ also merits investigation.

In this study, we successfully transformed $\mathrm{RCBr}$ using Agrobacterium vacuum infiltration and floral dip methods. We found that $\mathrm{RCBr}$ floral buds with a diameter less than $1 \mathrm{~mm}$ offer the most likely target for inoculation with Agrobacterium. A GFP marker was used in preference to kanamycin selection to identify candidate transformants. GFPpositive progenies were obtained from plants of three different ages via these two in planta methods. Transformation efficiencies were not significantly different among the treatment groups. PCR and Southern blot assays verified transformation, the availability of which opens numerous opportunities for expanded use of RCBr.

\section{Results}

\section{Selectable marker}

An appropriate selectable or screenable marker is a crucial component of genetic transformation protocols. In most plant transformation systems, an antibiotic or herbicide resistance gene or fluorescent protein gene is used. To identify a useful method for a rapid-cycling Brassica rapa (RCBr) transformation system, kanamycin resistance and green fluorescent protein (GFP) expression were tested on RcBC 1-33, the standard Wisconsin Fast Plants line of RCBr. Seeds were surface-sterilized and plated onto half strength MS media containing 4 different concentrations of kanamycin. After 12 days, green seedlings were still found in plates with $200 \mathrm{mg} / \mathrm{L}$ kanamycin, even when seeds were plated at a low density (Fig. 1). For GFP expression testing, sterilized wild-type seeds were plated onto half strength MS media. About 4 days later, over 100 seedlings were screened under a fluorescence stereomicroscope and none of them contained GFP signal (see Additional file 2). These results suggested that $\mathrm{RCBr}$ is relatively kanamycin-insensitive and that GFP would be a better selection marker than kanamycin resistance in a $\mathrm{RCBr}$ transformation system.

\section{Bud dissection to determine inoculation stage}

Because ovules are the target of Agrobacterium-mediated vacuum infiltration or floral dip transformation of Arabidopsis [21-23] and only young flowers with unclosed locules are transformable by these methods [23], flower bud dissection was performed to assess the $\mathrm{RCBr}$ bud growth stages most likely to yield successful transformants. Inflorescences with one or two open flowers were chosen and multiple floral buds were then dissected. Only buds with a diameter less than $1 \mathrm{~mm}$ had an unsealed carpel due to an

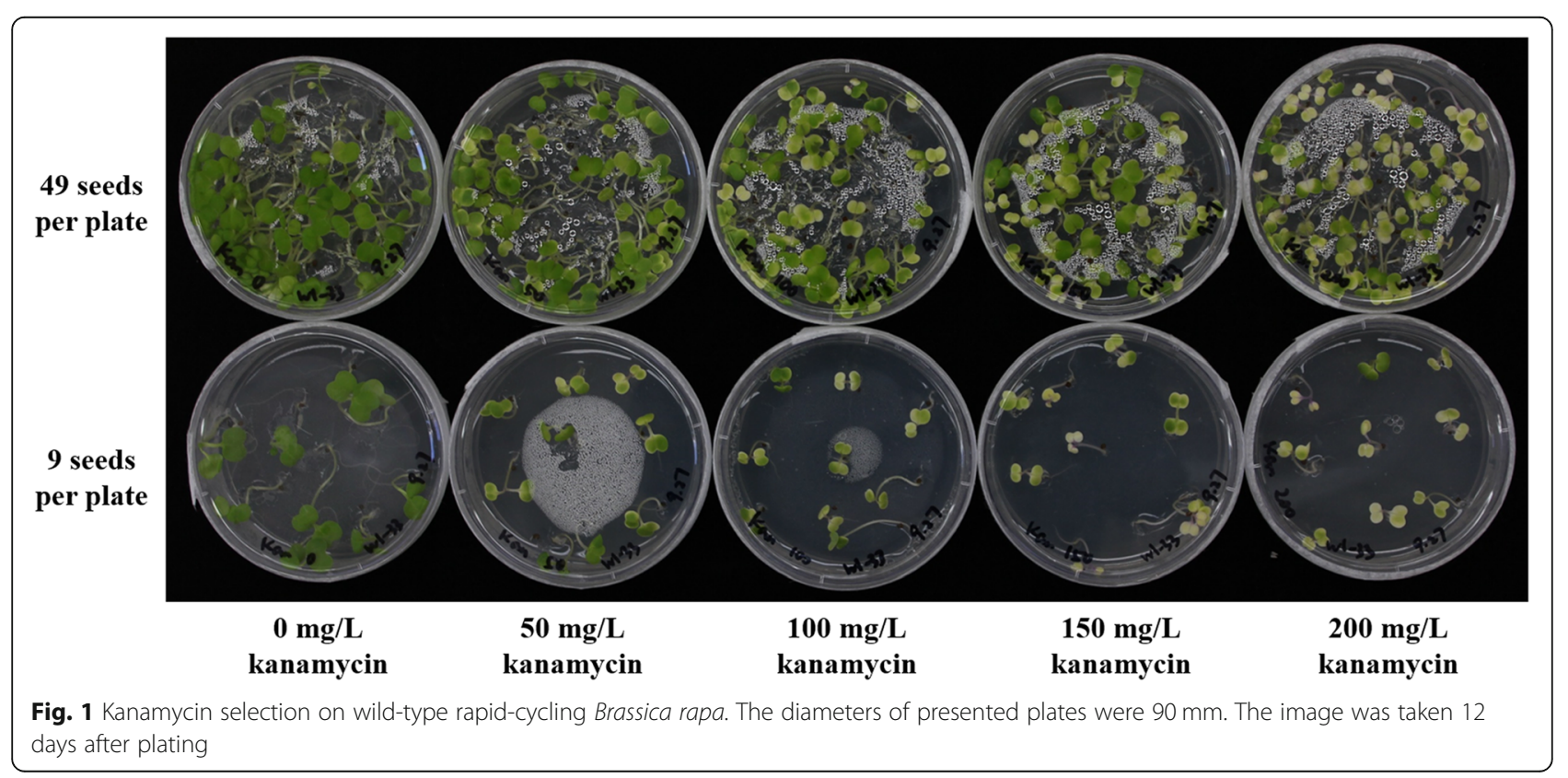


incompletely formed stigma, while locules of buds with diameters around $2 \mathrm{~mm}$ appeared to be closed (Fig. 2). Unlike Arabidopsis, only a limited subset of $\mathrm{RCBr}$ flowers form seed pods and application of Agrobacterium to flower buds with closed locules is likely to be unproductive [23]. As an added consideration, removing flowers or older buds requires additional labor and can destroy the whole inflorescence. The above findings suggested that application of Agrobacterium to $\mathrm{RCBr}$ might best be done at a relatively early stage of inflorescence development, for example when the oldest flower buds still have their petals mostly enclosed by sepals.

\section{Transformation by vacuum infiltration or floral dip}

Transformation of RCBr was attempted using both vacuum infiltration and floral dip approaches. Agrobacterium tumefaciens strain GV3101(pMP90) carrying binary plasmid p201G was utilized to deliver a 2X35S-GFP DNA construct. To test whether immature floral buds could be transformed and identify the best plant stage for Agrobacterium inoculation, different aged plants were tested. In the growth conditions used, 22-day-old plants were at a stage with recently opened flowers and immature buds of various ages (Fig. 3a). 14-day-old plants had larger unopened floral buds and buds $<1 \mathrm{~mm}$ in diameter (Fig. 3b), while 8-dayold seedlings were at a stage only containing the putative ideal buds $<1 \mathrm{~mm}$ in diameter (Fig. 3c). On the plants from these growth conditions that were more carefully examined, the $\sim 1 \mathrm{~mm}$ buds on 14-day-old plants were the 4th to 9th bud formed. Hence all plants carried buds of diameter less than $1 \mathrm{~mm}$ at the time of inoculation. However, floral dip was not attempted on 22-day-old plants because under our conditions the average number of pods formed per $\mathrm{RCBr}$ plant was around 4, but the buds predicted to be transformable on 22-day-old seedlings were 10th or 11th bud formed (Fig. 2). Due to their small size, vacuum infiltration was not used on 8-day-old seedlings. Seeds were harvested for all other treatments, generating a total of over 32,000 candidate $\mathrm{T}_{1}$ seeds across all treatments and treatment dates (Table 1).

$\mathrm{T}_{1}$ seedlings were subsequently screened using a fluorescence stereomicroscope 4-5 days after germination on half-strength MS media. GFP-positive plants were found in all four groups (Table 1; Fig. 4b). The results indicate that both the vacuum infiltration and floral dip methods can be used to transform $\mathrm{RCBr}$ via inoculation at multiple growth stages. No significant differences in transformation rate were observed between treatments in the present study (Fig. 4c).

\section{Confirmation of transformation}

$\mathrm{T}_{2}$ seedlings, from seeds that were obtained after selffertilization of GFP-positive $\mathrm{T}_{1}$ plants, were also screened for GFP signal using a fluorescence stereomicroscope. As expected for seed from hemizygous $\mathrm{T}_{1}$ plants, both GFPpositive and -negative plants were found among the $\mathrm{T}_{2}$

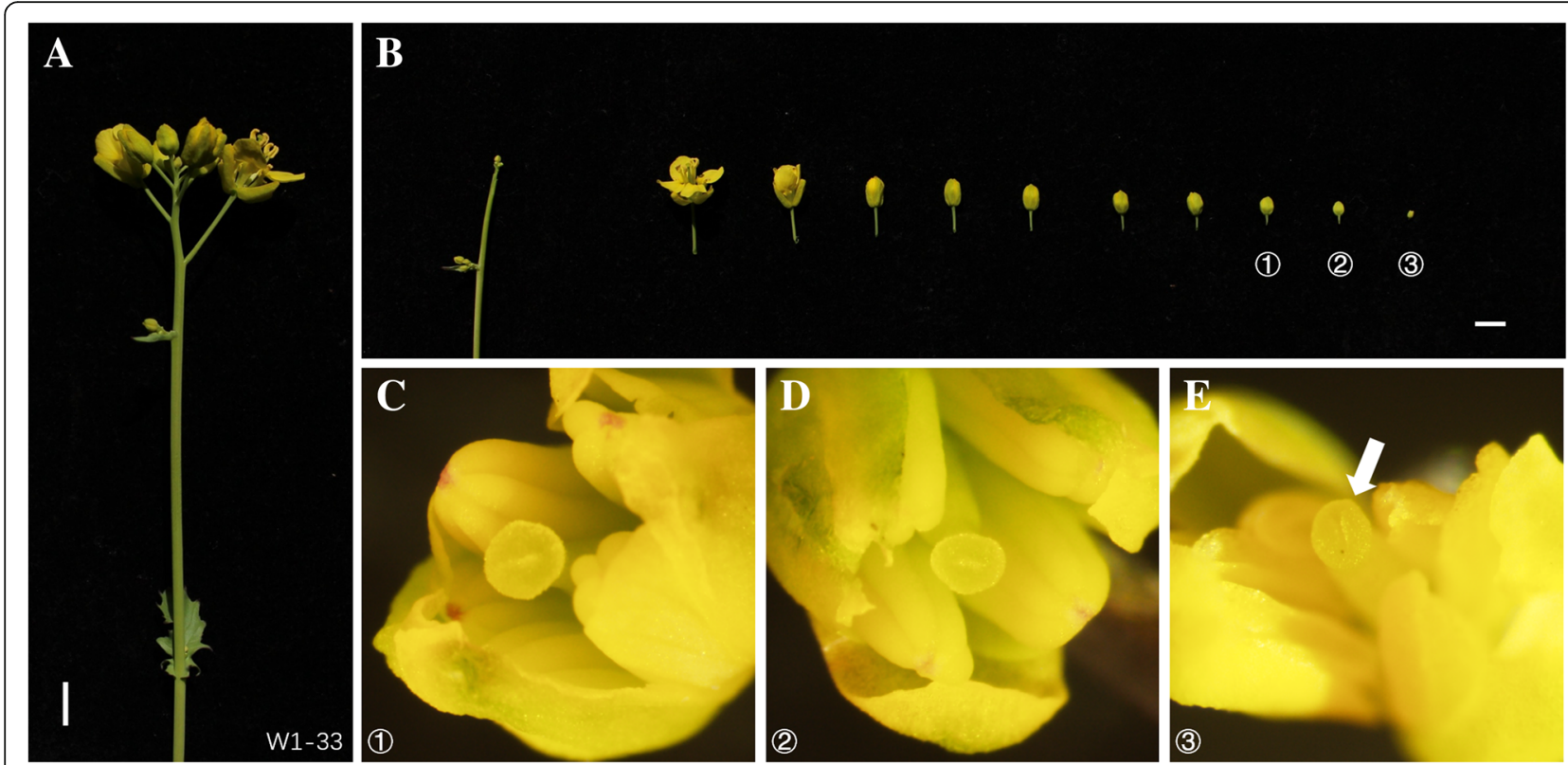

Fig. 2 Bud dissection of rapid-cycling Brassica rapa. a Intact inflorescence of RcBC 1-33, the standard line of RCBr, prior to dissection. b Floral buds from one inflorescence placed in order of occurrence on the stem. Scale bars $5 \mathrm{~mm}$. $\mathbf{c}$, $\mathbf{d}$ and $\mathbf{e}$, Dissection of indicated floral buds from B showing: (1) and (2), sealed carpels, diameters of buds were around $2 \mathrm{~mm}$. (3), unsealed carpel (white arrow), the diameter of bud was less than $1 \mathrm{~mm}$ 

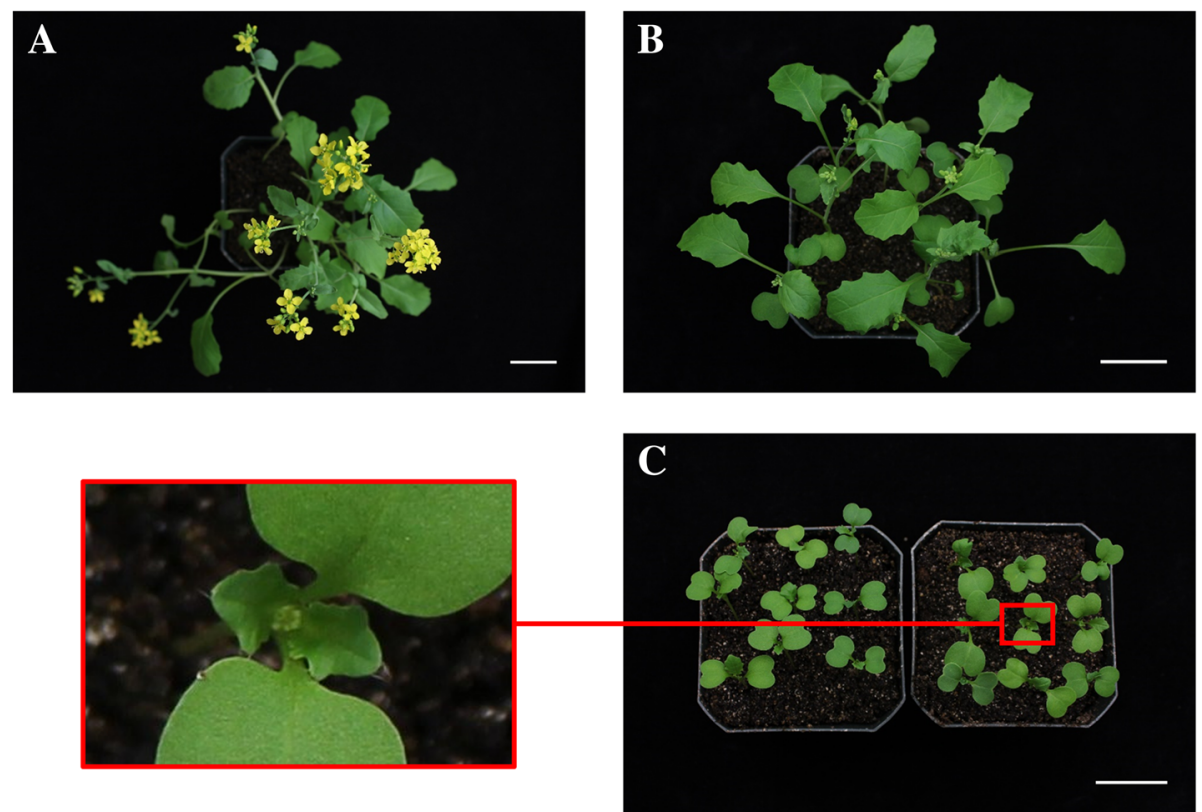

Fig. 3 Stages of rapid-cycling Brassica rapa chosen to transform. a 22-day-old seedlings. b 14-day-old seedlings. c 8-day-old seedlings. Scale bars, $3 \mathrm{~cm}$

progeny of GFP-positive $T_{1}$ plants. To further confirm genetic transformation, DNA was extracted from $\mathrm{T}_{2}$ plants and PCR and Southern blot assays were performed. Due to concerns that GFP fluorescence could arise from persistent Agrobacterium, four primer pairs were used for the PCR assays. Three of primer pairs target Agrobacterium DNAs not situated between the TDNA left border and right border (Fig. 5a). The "aph" and "backbone" primer pairs amplify binary plasmid sequences outside of the left and right borders respectively (Fig. 5a), while the "C58 glyA" primer pair amplifies a portion of the glyA gene on the A. tumefaciens chromosome [24]. PCR results showed that all GFP-positive $\mathrm{T}_{2}$ plants tested produced a GFP amplicon, while WT (non-inoculated) $\mathrm{RCBr}$ plants and $\mathrm{T}_{2}$ plants lacking green fluorescence were negative for the GFP amplicon (see for example Fig. $5 b)$. Importantly, none of the GFP-positive $T_{2}$ plants tested were positive for $A$. tumefaciens glyA signal. But surprisingly, over half of the plants tested gave a positive signal for the aph or backbone primer pairs, indicating persistent presence of DNA from outside of the T-DNA borders.

Samples from four $T_{2}$ plants that were GFP-positive by PCR assay, one GFP-negative $\mathrm{T}_{2}$ plant from a GFP-positive $\mathrm{T}_{1}$ plant, and one WT RCBr plant (non-inoculated) were also tested by Southern blot. Genomic DNA was digested with restriction enzyme $B a m H I$, separated by agarose gel electrophoresis, blotted and probed with a $720 \mathrm{bp}$ fragment of GFP (see Fig. 5a). Chromosomally integrated T-DNA appearing on the gel blot should be at least $1.4 \mathrm{~kb}$, and should vary in size depending on the variable distance to the next BamHI site, which would in many cases be provided by the flanking RCBr genome at the site of T-DNA integration (Fig. 5a). The observed minimal size of bands on the blot was about $1.5 \mathrm{~kb}$ (Fig. 5c). Multiple insertions were evident in the GFP-positive $\mathrm{T}_{2}$ plants (Fig. 5c). As is often observed after Agrobacterium-mediated plant transformation, some bands were observed that have shared size between independent transformants. These apparently result from intact T-DNA transfer beyond the left border to the next BamHI site in the vector (an expected $10.2 \mathrm{~kb}$ band for p201G), and from head-to-tail, tail-to-tail and other repetitive configurations of the integrated T-DNA. Importantly, in three of the four putative transformants, BamHI bands were also detected that were of unique size only to that transformant. The results provide strong evidence that the tested GFP-positive $T_{2}$ plants were genuine stable transformants.

In total, we obtained 33 GFP-positive $\mathrm{T}_{1}$ plants in the present study (Table 1). Ten of them were sterile or produced low quality seeds that did not germinate, while $T_{2}$ families were built from the other $23 \mathrm{~T}_{1}$ events. GFP screening results showed that all of these $T_{2}$ families contained GFP-positive plants. The $\mathrm{T}_{2}$ samples used in the PCR assay of Fig. $5 \mathrm{~b}$ were from 13 different $\mathrm{T}_{1}$ plants (FD6-1 and FD6-n1 were from the same $T_{1}$ plant). Hence evidence for the heritability of the transformation events, from $T_{1}$ transformant to $T_{2}$ progeny, 
Table 1 Transformation efficiencies for rapid-cycling Brassica rapa

\begin{tabular}{|c|c|c|c|c|}
\hline Method & Age of Seedlings (d) & Number of Seeds & Number of GFP-tagged T1 Seedlings & Transformation rate \\
\hline \multirow[t]{6}{*}{ Vacuum infiltration } & 22 & 1831 & 8 & $0.44 \%$ \\
\hline & & 763 & 0 & $0.00 \%$ \\
\hline & & 540 & 0 & $0.00 \%$ \\
\hline & & 759 & 0 & $0.00 \%$ \\
\hline & & 507 & 0 & $0.00 \%$ \\
\hline & & 768 & 1 & $0.13 \%$ \\
\hline \multirow[t]{3}{*}{ Vacuum infiltration } & 14 & 2065 & 2 & $0.10 \%$ \\
\hline & & 2669 & 4 & $0.15 \%$ \\
\hline & & 2928 & 6 & $0.20 \%$ \\
\hline \multirow[t]{10}{*}{ Floral dip } & 14 & 1437 & 0 & $0.00 \%$ \\
\hline & & 1295 & 2 & $0.15 \%$ \\
\hline & & 775 & 0 & $0.00 \%$ \\
\hline & & 745 & 1 & $0.13 \%$ \\
\hline & & 1653 & 0 & $0.00 \%$ \\
\hline & & 1307 & 1 & $0.08 \%$ \\
\hline & & 1019 & 1 & $0.10 \%$ \\
\hline & & 1585 & 0 & $0.00 \%$ \\
\hline & & 1348 & 2 & $0.15 \%$ \\
\hline & & 1640 & 0 & $0.00 \%$ \\
\hline \multirow[t]{6}{*}{ Floral dip } & 8 & 1621 & 1 & $0.06 \%$ \\
\hline & & 1722 & 1 & $0.06 \%$ \\
\hline & & 1712 & 3 & $0.18 \%$ \\
\hline & & 529 & 0 & $0.00 \%$ \\
\hline & & 934 & 0 & $0.00 \%$ \\
\hline & & 710 & 0 & $0.00 \%$ \\
\hline Overall & & 32,862 & 33 & $0.10 \%$ \\
\hline
\end{tabular}

was obtained for all of the GFP-positive $T_{1}$ plants that produced viable $T_{2}$ seed.

\section{Discussion}

Different genetic strains or subspecies of Brassica rapa exhibit strikingly different growth forms and uses (e.g., pakchoi, turnip, oilseed field mustard). Rapid-cycling Brassica rapa are very widely used in science education, and also have utility for scientific research. Genetic transformation of $\mathrm{RCBr}$ had not been reported. Although transformation methods that use tissue culture (transgenic callus production and plant regeneration) have been reported for Brassica rapa, they are difficult. Given past successes with in planta germline transformation not only with Arabidopsis but also with other Brassicaceae including Brassica rapa ssp. chinensis (pakchoi or bok choy), it was logical to attempt transformation of $\mathrm{RCBr}$ using vacuum infiltration or floral dip methods. Across all the treatment methods in this study, a transformation success rate of $0.1 \%$ was achieved (33 out of 32,862 candidate $T_{1}$ seeds tested). This is approximately ten-fold lower than the success rates commonly obtained with Arabidopsis but given the low amount of overall labor involved, it is well within range for a practically useful transformation method.

Kanamycin is widely used in plant transformation systems to select transformants, but high densities of seeds during selection have been shown to decrease the selective efficiency [25]. In our study, two different densities of wild-type $\mathrm{RCBr}$ were tested for kanamycin susceptibility and green seedlings were still found on plates even at very low densities using kanamycin at up to $200 \mathrm{mg} / \mathrm{L}$ (Fig. 1). This indicated that $\mathrm{RCBr}$ are less sensitive to kanamycin. Takasaki et al. [26] used Agrobacterium-mediated tissue culture to transform Brassica rapa ssp. chinensis and could not avoid escaped non-transgenic shoots using 50 mg/L kanamycin selection. Kuvshinov et al. [27] had a similar issue while transforming Brassica rapa ssp. oleifera, that the portion of escaped shoots was $90 \%$ under kanamycin selection. However, explant tissue of Brassica rapa showed acceptable sensitivity to kanamycin [28]. These studies suggest 


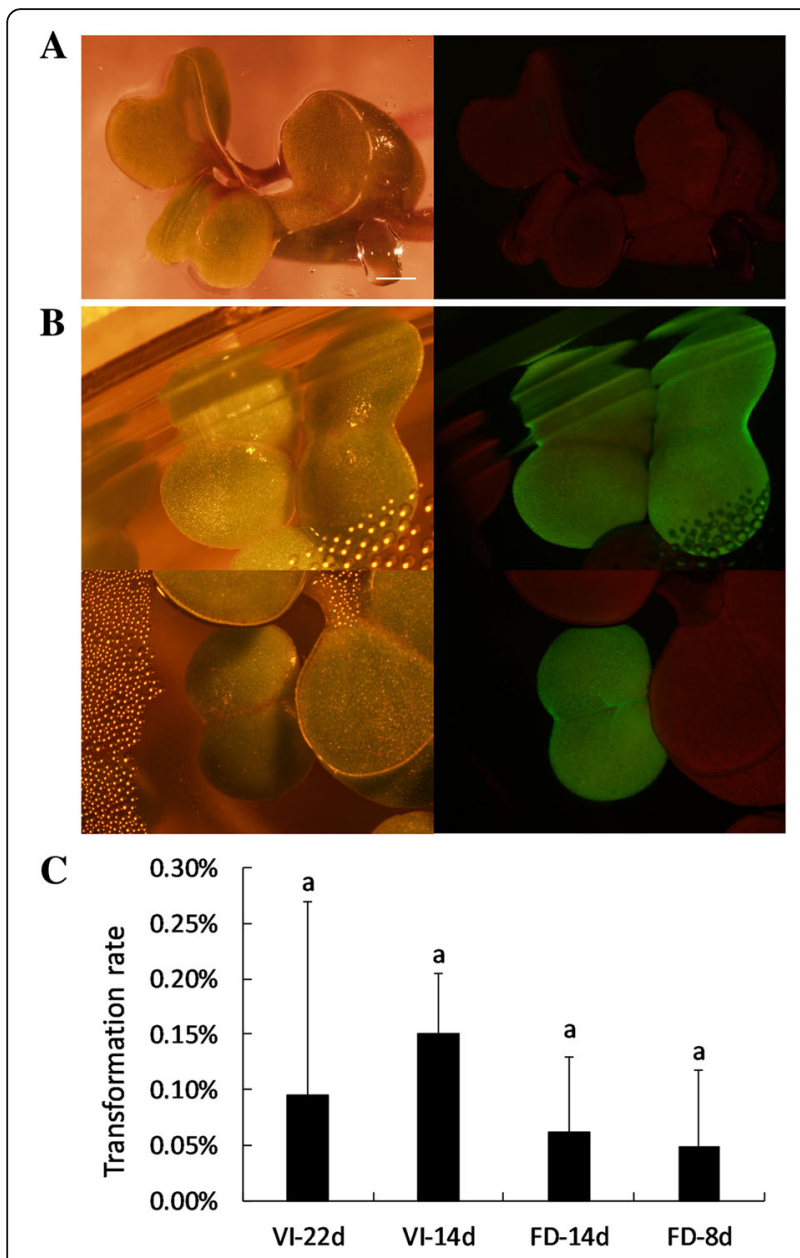

Fig. 4 Transformation of rapid-cycling Brassica rapa. a An example of GFP-negative seedlings. Scale bar, $1 \mathrm{~mm}$. b Two examples of GFPpositive $T_{1}$ seedlings. c Transformation efficiencies for the different treatments. Mean +/- SE for the data in Table 1. The means are not significantly different (ANOVA, $p<0.05$ ). VI, vacuum infiltration; FD, floral dip

that the kanamycin sensitivities among Brassica rapa plants are variable. Although GFP worked well in our RCBr transformation system, it is a screenable marker that requires researchers to be the "selective" agent. Considering this aspect, other selectable markers could be tested on $\mathrm{RCBr}$ in future studies.

Ovules are known to be the primary target of Agrobacterium-mediated vacuum infiltration and floral dip transformation in Arabidopsis [21-23]. Providing Agrobacterium with ready access to developing ovules in the locule interior is likely to be a key aspect for success with in planta transformation of other plants as well. Desfeux et al. [23] found that transformation failed with floral buds inoculated past the stage at which the stigmatic cap closes the locule, and found that the rate of transformation of an Arabidopsis CRABS-CLAW mutant that has unsealed carpels was 6fold higher than the transformation rate of wild-type plants.
Dissection work was executed in our study to identify buds with unclosed carpels. We found that the diameter of such buds was less than $1 \mathrm{~mm}$ (Fig. 2), which was similar to the result that the pakchoi flowers with GUSstained ovules after Agrobacterium inoculation were about $0.5-1.0 \mathrm{~mm}$ in diameter and had an open ovary when vacuum infiltration was carried out [15]. Although Xu et al. [15] found GUS stained pollen in the same sample with stained ovules, they did not determine if either or both were the source of successfully transgenic plants. In Arabidopsis, Ye et al. [21] found mature pollen expressing GUS after Agrobacterium infiltration, while Desfeux et al. [23] did not find such pollen after floral dipping. However, neither of them could get transformants from crossing when inoculated plants served as pollen donors and noninoculated plants served as pollen recipients [21, 23]. Recently, it was reported that prairie gentian (Eustoma grandifloru; also called lisianthus) was successfully transformed via the floral dip method at a post-anthesis stage when ovaries were partially sealed, while in these flowers a stylar channel remained open [18]. Another study recently reported tomato transformation by floral bud injection [17], another approach to providing Agrobacterium with access to ovules. We took care to only inoculate plants carrying immature flower buds $(<1 \mathrm{~mm})$, but were surprised that we did not observe significant differences in transformation frequency between plants with only immature buds or plants already carrying open flowers as well as immature buds. Additional studies in multiple laboratories will be required to determine if transformation rates are more favorable for certain plant growth stages or other treatment regimes. In practical terms, the fecundity of plants is another factor influencing the transformation success, especially for self-infertile accessions.

With Agrobacterium mediated transformation using binary vectors it is common that backbone sequences (DNA outside of the left and right borders) can be integrated into the plant genome [29]. The frequencies of this phenomenon were over $60 \%$ in transgenic tobacco [30], strawberry [31] and wheat [32]. PCR analysis from our research presented a similar result that many of the examined plants had backbone sequence integration events (Fig. 5b). Strikingly, the entire vector sequence can be incorporated into the plant DNA [33, 34]. This apparently happened in our study as well, as we observed multiple $T_{2}$ plants that were PCR-positive for all three of the vector sequence primer pairs (GFP, aph and backbone). Southern blot evidence similarly showed some T-DNAs that extended past the left border to include the full $10.2 \mathrm{~kb}$ BamHI fragment of the p201G vector (Fig. 5c). The Southern blot lanes shared other bands among transformants, especially between entirely independent 


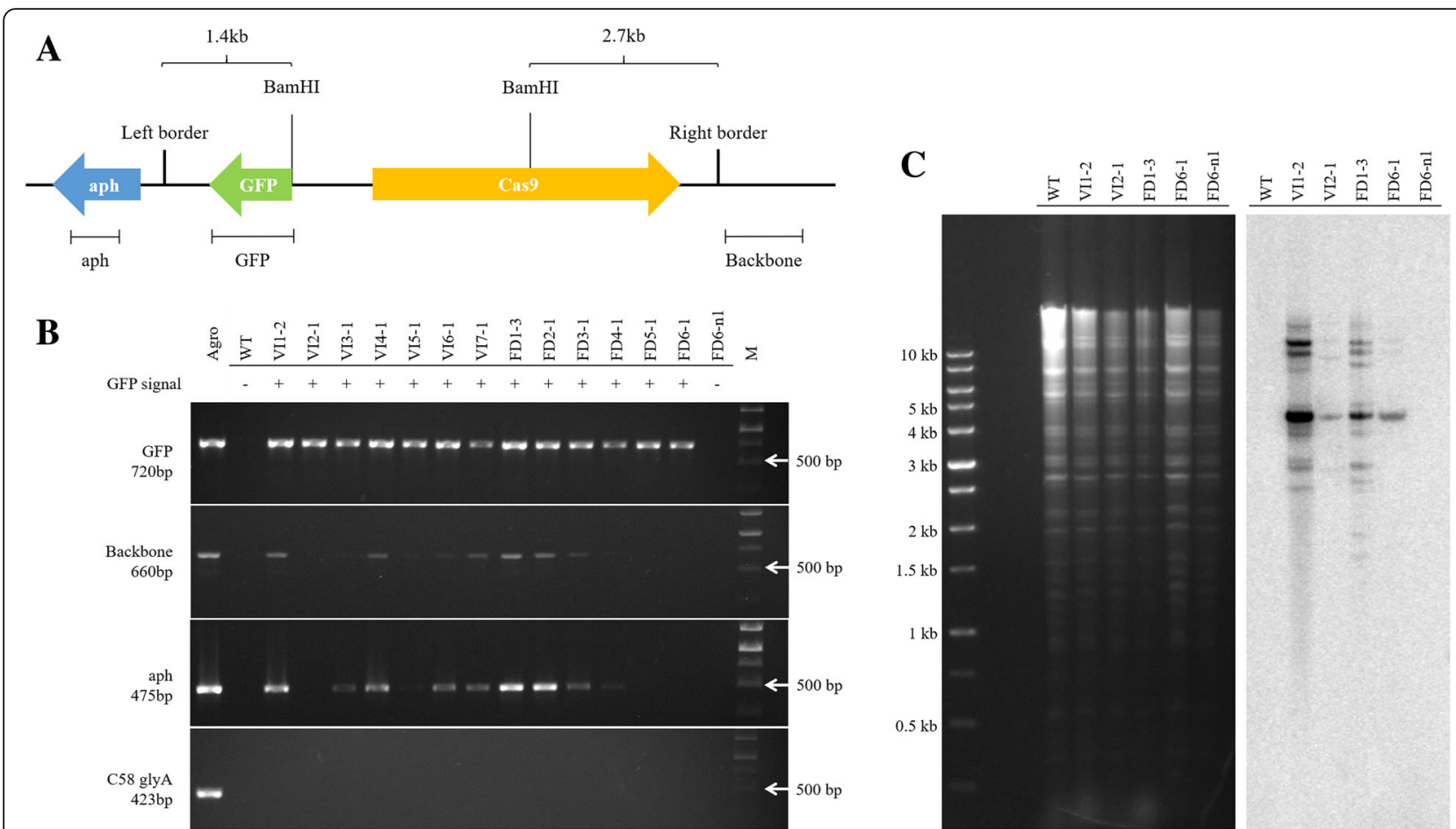

Fig. 5 Verification of rapid-cycling Brassica rapa transformation. a Schematic of T-DNA and flanking region of the circular binary vector p201G, with corresponding aph, GFP and backbone PCR products depicted at bottom. $\mathbf{b}$ representative results of PCR assays; +, GFP-positive; -, GFPnegative; Agro, Agrobacterium strain GV3101(pMP90)(p201G); WT, wild-type RCBr plant; VI, $T_{2}$ from vacuum infiltration; FD, $T_{2}$ from floral dip; M, marker. C58 glyA is an Agrobacterium chromosomal gene. c Southern blot analysis. Left, electrophoresis gel of BamHI-digested plant genomic DNA; right, Southern blot probed with GFP probe indicated in (a)

VI1-2 and FD1-3, suggesting that direct or inverted T-DNA repeats had been integrated into one locus, while the bands of unique size revealed independent insertions (Fig. 5c). Multicopy insertions, which have the potential to cause transgene silencing [35], are often less desirable. Most of the tested GFP-positive plants in our study had this issue. To address the problem, Oltmanns et al. [36] have suggested launching T-DNA from the Agrobacterium chromosome, which could also reduce the backbone sequence integration. De Paepe et al. [37] reported that using a T-DNA vector with one $\operatorname{lox} P$ site to transform $C R E$-expressing Arabidopsis would increase the frequency of single-copy events. Arabidopsis researchers have reduced the frequency of multicopy inserts by switching to less efficient Agrobacterium strains. These and other refinements remain to be explored in $\mathrm{RCBr}$, for which successful vacuum infiltration and floral dip transformation have now been demonstrated.

\section{Conclusion}

A transformation system for $\mathrm{RCBr}$ was established in this study. This will promote development of new biology teaching tools as well as basic biology research on Brassica rapa.

\section{Methods}

Plant growth and pollination

Rapid-cycling Brassica rapa seeds (standard line, RcBC 1-33, available from the Rapid-Cycling Brassica Collection, University of Wisconsin - Madison, www.rcbc.wisc.edu) were kindly provided by Dr. Paul Williams (University of Wisconsin Madison, USA). Seeds were sown 9 per pot in a soil mixture of Sun Gro $^{\circ}$ (Sun Gro Horticultural, Agawam, MA, USA) propagation mix and vermiculite (1:1). For vacuum infiltration experiments, pots were covered with tulle fabric (plastic mesh with pore diameter approximately $1 \mathrm{~mm}$ ) at planting, to prevent soil from falling into Agrobacterium inoculum during infiltration. Plants were grown at $24^{\circ} \mathrm{C}$ under a $16-\mathrm{h}$ light/8-h dark photoperiod. After inoculation with Agrobacterium, flowers of $\mathrm{T}_{0}$ plants were manually cross-fertilized every day (for at least 1 week) on the most recently opened flowers, using a bee-stick (the body of a dead bee glued to a toothpick) carrying mixed pollen from multiple inoculated RCBr plants. Self-fertilization was performed on buds of $\mathrm{T}_{1}$ plants to generate $T_{2}$ seeds. For self-fertilization, forceps were used to remove sepals and petals and expose the young stigma on newly developing flowers. Stamens were collected from opened flowers of the same plant and used for pollination. Forceps were soaked in $70 \%$ ethanol before each selffertilization to avoid pollen contamination. 
Agrobacterium tumefaciens strain, culture and inoculation Agrobacterium tumefaciens strain GV3101(pMP90) carrying binary vector p201G was used in all experiments [38]. p201G carries a 2X35S promoter-mGFP5-nos terminator gene construct [39]. Agrobacterium was diluted in LB medium (1:100) with $25 \mathrm{mg} / \mathrm{L}$ kanamycin and $50 \mathrm{mg} / \mathrm{L}$ rifampicin, and cultured overnight at $28{ }^{\circ} \mathrm{C}, 200 \mathrm{rpm}$. Bacteria were collected by centrifugation at $5000 \mathrm{rpm}$ for 10 min at room temperature and resuspended with 5\% sucrose and $200 \mu \mathrm{L} / \mathrm{L}$ Silwet L-77 to a final $\mathrm{OD}_{600}$ of $0.8-$ 1.0. Vacuum infiltration or floral dip were performed following the procedure described by Clough and Bent [20]. For vacuum infiltration, at least $800 \mathrm{~mL}$ of inoculum was used. Above-soil portions of plants were inverted into the Agrobacterium solution in a desiccator jar which was then attached to a vacuum pump via rubber tube. A vacuum was established in the desiccator jar until the infiltration solution had formed bubbles for approximately $10 \mathrm{~s}$, at which time the desiccator jar was opened to atmospheric pressure by removal of the rubber tube, causing infiltration of the Agrobacterium solution into the immersed plant tissues. For floral dip, about $3 \mathrm{~mL}$ of Agrobacterium solution was used by pipetting onto multiple flower buds. After either form of inoculation, plants were placed in a plastic tray and covered by a plastic dome to maintain elevated humidity. Plants were then maintained in a lowlight environment for $16-24 \mathrm{~h}$ and then uncovered and returned to the growth chamber in which they had previously been cultivated. For each treatment, more than 20 plants were inoculated.

\section{Bud dissection}

Flowers and buds were taken from the same inflorescence. Fine forceps were used to separate sepals and petals. A stereomicroscope was employed to look for unsealed carpels where the stigma had not fully formed. Photos were taken with an attached Olympus DP73 camera. Predissection diameters were measured by Fiji software.

\section{Kanamycin sensitivity testing or GFP screening}

$\mathrm{RCBr}$ seeds were surface-sterilized for around $20 \mathrm{~h}$ under chlorine gas using the vapor-phase method [20]. For kanamycin sensitivity testing, surface-sterilized wild-type seeds were sown onto half strength Murashige and Skoog agar media containing $0,50,100,150$ or $200 \mathrm{mg} / \mathrm{L}$ kanamycin. The densities were 49 or 9 seeds per plate. For GFP screening, sterilized seeds were plated onto half strength MS media without antibiotic agents. Seedlings were grown in the same environment described above. To detect GFP signal, 4- to 7-day-old seedlings were screened using a Leica MZ FLIII fluorescence stereomicroscope with GFP2 (GFP plus) filter. Photos were taken with an attached Olympus DP73 camera.

\section{PCR and Southern blots}

Total genomic DNA of $\mathrm{T}_{2}$ plants was isolated via a CTAB method. GoTaq ${ }^{\bullet}$ Green Master Mix (Promega) was used in PCR assays. Primers are listed in Additional file 1 . The PCR program included pre-denaturation at $95^{\circ} \mathrm{C}$ for $3 \mathrm{~min}$, followed by 30 cycles of denaturing at $95^{\circ} \mathrm{C}$ for $30 \mathrm{~s}$, annealing at $57^{\circ} \mathrm{C}$ for $30 \mathrm{~s}$ and extension at $72{ }^{\circ} \mathrm{C}$ for $45 \mathrm{~s}$, and a final extension at $72{ }^{\circ} \mathrm{C}$ for $5 \mathrm{~min}$. The products were analyzed by electrophoresis in $1.0 \%$ agarose-ethidium bromide gels.

Southern blot experiments were conducted as in Current Protocols in Molecular Biology [40]. Approximately $10 \mu \mathrm{g}$ of genomic DNA of each sample was digested with the restriction enzyme $B a m \mathrm{HI}$, resolved by electrophoresis in a $0.7 \%$ agarose gel, transferred to a Hybond $^{\mathrm{TM}}-\mathrm{N}$ membrane, crosslinked by baking at $80^{\circ} \mathrm{C}$ for $2 \mathrm{~h}$. Blots were then probed with a ${ }^{32} \mathrm{P}$-labeled GFP gene fragment (Additional file 1) and imaged using a Typhoon $^{\mathrm{TM}}$ FLA 9000 gel imaging scanner.

\section{Additional files}

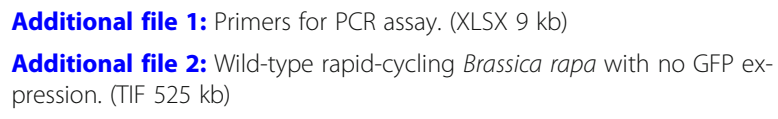

\section{Abbreviations}

FD: Floral dip; GFP: Green fluorescent protein; RCBr: Rapid-cycling of Brassica rapa; VI: Vacuum infiltration

\section{Acknowledgments}

The authors thank Dr. Paul Williams for providing RCBr seeds and helpful discussions, and Dr. Jinwoo Bok and Dr. Nancy Keller for their support of Southern blot experiments. The experiments were performed at University of Wisconsin - Madison.

Authors' contributions

$\mathrm{DH}, \mathrm{AB}$, and $\mathrm{YL}$ designed the research. $\mathrm{DH}$ performed the experiments, analyzed the data and wrote the manuscript. $\mathrm{DH}, \mathrm{AB}$, and $\mathrm{YL}$ contributed to interpreting the results. $\mathrm{DH}, \mathrm{AB}, \mathrm{XH}$ and $\mathrm{YL}$ read, edited and approved the final manuscript.

\section{Funding}

DH was supported by a scholarship from the China Scholarship Council. The research was supported by the National Natural Science Foundation of China (31872106 and 31471886) and National Vegetable Industry Technology System (CARS-23-A-06). The funding agencies had no role in research design, data collection and analysis, or manuscript writing.

\section{Availability of data and materials}

All data generated or analysed during this study are included in this published article.

Ethics approval and consent to participate Not applicable.

\section{Consent for publication}

Not applicable.

Competing interests

The authors declare that they have no competing interests. 
Received: 12 February 2019 Accepted: 21 May 2019

Published online: 10 June 2019

\section{References}

1. Williams PH, Hill CB. Rapid-cycling populations of Brassica. Science. 1986;232:1385-9.

2. Wisconsin Fast Plants of the University of Wisconsin. https://fastplants.org/. Accessed 3 Dec 2018.

3. Gervasi DDL, Schiestl FP. Real-time divergent evolution in plants driven by pollinators. Nat Commun. 2017:8:14691.

4. Lin K, Zhang N, Severing El, Nijveen H, Cheng F, Visser RG, et al. Beyond genomic variation - comparison and functional annotation of three Brassica rapa genomes: a turnip, a rapid cycling and a Chinese cabbage. BMC Genomics. 2014;15:250

5. Iniguez-Luy FL, Lukens L, Farnham MW, Amasino RM, Osborn TC. Development of public immortal mapping populations, molecular markers and linkage maps for rapid cycling Brassica rapa and B. oleracea. Theor App Genet. 2009:120:31-43.

6. Lascoux M, Lee JK. One step beyond lethal equivalents: characterization of deleterious loci in the rapid cycling Brassica rapa L. base population. Genetica. 1998;104:161-70.

7. Rood SB, Williams PH, Pearce D, Murofushi N, Mander LN, Pharis RP. A mutant gene that increases gibberellin production in Brassica. Plant Physiol. 1990:93:1168-74

8. Murata M, Orton TJ. Callus initiation and regeneration capacities in Brassica species. Plant Cell Tissue Organ Cult. 1987;11:111-23.

9. Li G, Yue L, Li F, Zhang S, Zhang H, Qian W, et al. Research progress on Agrobacterium tumefaciens-based transgenic technology in Brassica rapa. Hortic Plant J. 2018:4:126-32

10. Teo W, Lakshmanan P, Kumar P, Goh C-J, Swarup S. Direct shoot formation and plant regeneration from cotyledon explants of rapid-cycling Brassica rapa. In Vitro Cell Dev Biol Plant. 1997;33:288-92.

11. Cogbill S, Faulcon T, Jones G, McDaniel M, Harmon G, Blackmon R, et al. Adventitious shoot regeneration from cotyledonary explants of rapid-cycling fast plants of Brassica rapa L. Plant Cell Tissue Organ Cult. 2010;101:127-33.

12. Herrera Díaz A. Regeneration and plastid transformation approaches in Arabidopsis thaliana and rapid-cycling Brassica rapa. München: LMU; 2011.

13. Bechtold N, Ellis J, Pelletier G. In planta Agrobacterium-mediated gene transfer by infiltration of adult Arabidopsis thaliana plants. C R Acad Sci Paris Life Sci. 1993;316:1194-9.

14. Bent A. Arabidopsis in planta transformation. Uses, mechanisms, and prospects for transformation of other species. Plant Physiol. 2000;124: $1540-7$

15. Xu H, Wang X, Zhao H, Liu F. An intensive understanding of vacuum infiltration transformation of pakchoi (Brassica rapa ssp. chinensis). Plant Cell Rep. 2008;27:1369-76.

16. Saha P, Blumwald E. Spike-dip transformation of Setaria viridis. Plant J. 2016; 86:89-101

17. Satya Sharada M, Kumari A, Kumar Pandey A, Sharma S, Sharma P, Sreelakshmi Y, et al. Generation of genetically stable transformants by Agrobacterium using tomato floral buds. Plant Cell Tissue Organ Cult. 2017; 129:299-312.

18. Fang F, Oliva M, Ehi-Eromosele S, Zaccai M, Arazi T, Oren-Shamir M. Successful floral-dipping transformation of post-anthesis lisianthus (Eustoma grandiflorum) flowers. Plant J. 2018:96:869-79.

19. Cao MQ, Liu F, Yao L, Bouchez D, Tourneur C, Li Y, et al. Transformation of pakchoi (Brassica rapa L. ssp chinensis) by Agrobacterium infiltration. Mol Breed. 2000;6:67-72

20. Clough SJ, Bent AF. Floral dip: a simplified method for Agrobacteriummediated transformation of Arabidopsis thaliana. Plant J. 1998:16:735-43.

21. Ye G-N, Stone D, Pang S-Z, Creely W, Gonzalez K, Hinchee M. Arabidopsis ovule is the target for Agrobacterium in planta vacuum infiltration transformation. Plant J. 1999;19:249-57.

22. Bechtold N, Jaudeau B, Jolivet S, Maba B, Vezon D, Voisin R, et al. The maternal chromosome set is the target of the T-DNA in the in planta transformation of Arabidopsis thaliana. Genetics. 2000;155:1875-87.

23. Desfeux C, Clough SJ, Bent AF. Female reproductive tissues are the primary target of Agrobacterium-mediated transformation by the Arabidopsis floral-dip method. Plant Physiol. 2000;123:895-904.
24. Deeba F, Hyder M, Shah S, Naqvi SM. Multiplex PCR assay for identification of commonly used disarmed Agrobacterium tumefaciens strains. Springerplus. 2014;3:358.

25. Nagashima $\mathrm{Y}$, Koiwa $\mathrm{H}$. High throughput selection of antibiotic-resistant transgenic Arabidopsis plants. Anal Biochem. 2017;525:44-5.

26. Takasaki T, Hatakeyama K, Ojima K, Watanabe M, Toriyama K, Hinata K. Factors influencing Agrobacterium-mediated transformation of Brassica rapa L. Breed Sci. 1997;47:127-34.

27. Kuvshinov V, Koivu K, Kanerva A, Pehu E. Agrobacterium tumefaciens-mediated transformation of greenhouse-grown Brassica rapa ssp. oleifera. Plant Cell Rep. 1999:18:773-7.

28. Radke S, Turner J, Facciotti D. Transformation and regeneration of Brassica rapa using Agrobacterium tumefaciens. Plant Cell Rep. 1992;11:499-505.

29. Lee L-Y, Gelvin SB. T-DNA binary vectors and systems. Plant Physiol. 2008; 146:325-32.

30. Kononov ME, Bassuner B, Gelvin SB. Integration of T-DNA binary vector "backbone" sequences into the tobacco genome: evidence for multiple complex patterns of integration. Plant J. 1997;11:945-57.

31. Abdal-Aziz SA, Pliego-Alfaro F, Quesada MA, Mercado JA. Evidence of frequent integration of non-T-DNA vector backbone sequences in transgenic strawberry plant. J Biosci Bioeng. 2006;101:508-10.

32. Wu H, Sparks CA, Jones HD. Characterisation of T-DNA loci and vector backbone sequences in transgenic wheat produced by Agrobacterium-mediated transformation. Mol Breed. 2006;18:195-208.

33. Wenck A, Czakó M, Kanevski I, Márton L. Frequent collinear long transfer of DNA inclusive of the whole binary vector during Agrobacterium-mediated transformation. Plant Mol Biol. 1997;34:913-22.

34. De Buck S, De Wilde C, Van Montagu M, Depicker A. T-DNA vector backbone sequences are frequently integrated into the genome of transgenic plants obtained by Agrobacterium-mediated transformation. Mol Breed. 2000;6:459-68.

35. Gelvin SB. Integration of Agrobacterium T-DNA into the plant genome. Annu Rev Genet. 2017:51:195-217.

36. Oltmanns H, Frame B, Lee L-Y, Johnson S, Li B, Wang K, et al. Generation of backbone-free, low transgene copy plants by launching T-DNA from the Agrobacterium chromosome. Plant Physiol. 2010;152:1158-66.

37. De Paepe A, De Buck S, Hoorelbeke K, Nolf J, Peck I, Depicker A. High frequency of single-copy T-DNA transformants produced by floral dip in CRE-expressing Arabidopsis plants. Plant J. 2009:59:517-27.

38. Koncz C, Schell J. The promoter of $T_{L}$-DNA gene 5 controls the tissuespecific expression of chimaeric genes carried by a novel type of Agrobacterium binary vector. Mol Gen Genet. 1986;204:383-96.

39. Jacobs TB, LaFayette PR, Schmitz RJ, Parrott WA. Targeted genome modifications in soybean with CRISPR/Cas9. BMC Biotechnol. 2015;15:16.

40. Ausubel FM, Brent R, Kingston RE, Moore DD, Seidman JG, Smith JA, et al. Current protocols in molecular biology. New York: Wiley; 1997.

\section{Publisher's Note}

Springer Nature remains neutral with regard to jurisdictional claims in published maps and institutional affiliations.

Ready to submit your research? Choose BMC and benefit from:

- fast, convenient online submission

- thorough peer review by experienced researchers in your field

- rapid publication on acceptance

- support for research data, including large and complex data types

- gold Open Access which fosters wider collaboration and increased citations

- maximum visibility for your research: over $100 \mathrm{M}$ website views per year

At BMC, research is always in progress.

Learn more biomedcentral.com/submissions 